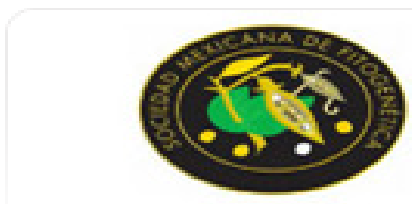

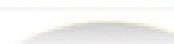

Revista Fitotecnia Mexicana

ISSN: 0187-7380

revfitotecniamex@gmail.com

Sociedad Mexicana de Fitogenética, A.C.

México

López-Salinas, Ernesto; Tosquy-Valle, Óscar H.; Villar-Sánchez, Bernardo; Acosta-

Gallegos, Jorge A.; Rodríguez-Rodríguez, José R.; Andrés-Meza, Pablo

RENDIMIENTO Y ESTABILIDAD DE LÍNEAS MEJORADAS DE FRIJOL NEGRO EN

VERACRUZ Y CHIAPAS, MÉXICO

Revista Fitotecnia Mexicana, vol. 38, núm. 2, 2015, pp. 173-181

Sociedad Mexicana de Fitogenética, A.C.

Chapingo, México

Disponible en: http://www.redalyc.org/articulo.oa?id=61038806007

- Cómo citar el artículo

- Número completo

- Más información del artículo

- Página de la revista en redalyc.org

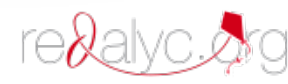

Sistema de Información Científica

Red de Revistas Científicas de América Latina, el Caribe, España y Portugal Proyecto académico sin fines de lucro, desarrollado bajo la iniciativa de acceso abierto 


\title{
RENDIMIENTO Y ESTABILIDAD DE LÍNEAS MEJORADAS DE FRIJOL NEGRO EN VERACRUZ Y CHIAPAS, MÉXICO
}

\author{
YIELD AND STABILITY OF IMPROVED LINES OF BLACK BEAN \\ IN VERACRUZ AND CHIAPAS, MÉXICO
}

\author{
Ernesto López-Salinas' ${ }^{1}$, Oscar H. Tosquy-Valle ${ }^{1}$, Bernardo Villar-Sánchez ${ }^{2}$, \\ Jorge A. Acosta-Gallegos ${ }^{3 *}$, José R. Rodríguez-Rodríguez ${ }^{4}$ y Pablo Andrés-Meza ${ }^{5}$
}

\begin{abstract}
${ }^{1}$ Campo Experimental Cotaxtla, Instituto Nacional de Investigaciones Forestales, Agrícolas y Pecuarias (INIFAP). Km 34.5 Carr. Federal Veracruz-Córdoba. Apartado Postal 429. 91700, Veracruz, Ver. ${ }^{2}$ Campo Experimental Centro de Chiapas, INIFAP. Km 3.0 Carr. Ocozocoautla-Cintalapa. 29140, Ocozocoautla, Chis. ${ }^{3}$ Campo Experimental Bajío, INIFAP. Km 6.5 Carr. Celaya-San Miguel de Allende. 38110, Celaya, Gto. ${ }^{4}$ Campo Experimental Ixtacuaco, INIFAP. Km 4.5 Carr. Federal Martínez de la Torre-Tlapacoyan. Apartado Postal 162. 93600, Martínez de la Torre, Ver. ${ }^{5}$ Campus Montecillo, Colegio de Postgraduados. Km 36.5 Carr. México-Texcoco. 56230, Montecillo, Texcoco, Estado de México.
\end{abstract}

*Autor para correspondencia (acosta.jorge@inifap.gob.mx)

\section{RESUMEN}

El análisis de la interacción genotipo por ambiente permite seleccionar genotipos estables, de amplia adaptación y rendimiento superior; así como identificar ambientes en los cuales la interacción con los genotipos sea menor. En los años 2011 y 2012 se sembró un ensayo de rendimiento conformado por 10 líneas avanzadas y dos testigos comerciales de frijol negro (Phaseolus vulgaris L.) en 12 combinaciones de localidades y sistemas de producción en los estados de Veracruz y Chiapas, México. En cada localidad se utilizó un diseño de bloques completos al azar con cuatro repeticiones. Los datos de rendimiento se sometieron a análisis de varianza individuales por ambiente y combinado. Para clasificar los ambientes e identificar líneas sobresalientes por su rendimiento de grano y menor interacción con el ambiente, se utilizó el modelo de efectos principales aditivos e interacción multiplicativa (AMMI). De acuerdo con análisis AMMI, el efecto ambiental fue mayor que el de genotipos; las localidades de Martínez de la Torre y San Andrés Tuxtla mostraron menor interacción y se consideran adecuadas para realizar selección en el proceso de mejoramiento. En algunos ambientes la incidencia de enfermedades disminuyó el rendimiento de los genotipos susceptibles y contribuyó al efecto ambiental $\mathrm{y}$ a la interacción genotipo $\mathrm{x}$ ambiente. En seis de los 12 ambientes de prueba los testigos fueron significativamente (P $<0.05$ ) superados por la mejor línea, que fue superior en seis de los 12 ambientes de prueba. Las líneas NGO 07022 y SCN 2 mostraron baja interacción con los ambientes y estuvieron en el grupo de mayor rendimiento. La línea SEN 70 obtuvo el mayor rendimiento promedio (1437 $\left.\mathrm{kg} \mathrm{ha}^{-1}\right)$, superior a los testigos Papaloapan y Comapa en 9 y 7 $\%$, respectivamente, pero mostró alta interacción con el ambiente, con adaptación específica en los ambientes de menor rendimiento.

Palabras clave: Phaseolus vulgaris, enfermedades, interacción genotipo $\mathrm{x}$ ambiente.

\section{SUMMARY}

The analysis of the interaction genotype by environment allows for selection of stable genotypes, of wide adaptation and superior yield, as well as identification of environments of small genotype by environment interaction. During the years 2011 and 2012, a yield trial made up of 10 advanced lines and two commercial cultivars of black seeded common bean (Phaseolus vulgaris L.) was sown in 12 site-production system combinations in the states of Veracruz and Chiapas, México. At each environment the trial was sown under a random complete block design with four replications. Yield data were analyzed per individual trial and in a combined analysis. The model of additive main effects and multiplicative interaction (AMMI) was used to classify the environments and to identify outstanding high yielding lines with low interaction with the environment. According to AMMI analysis, the environmental effect was greater than that of the genotypes; sites Martínez de la Torre and San Andrés Tuxtla showed low interaction and thus are considered suitable for selection in the breeding process. In some environments, the incidence of diseases limited seed yield of susceptible genotypes and contributed towards the effects of environment and genotype by environment interaction. Control cultivars were significantly $(\mathrm{P}<0.05)$ out-yielded by a line in six of the 12 test environments. Lines NGO 07022 and SCN 2 showed low interaction with the environments and were in the high yielding group. Line SEN 70 displayed the highest average yield of $1437 \mathrm{~kg} \mathrm{ha}^{-1}$, thus out yielding Papaloapan and Comapa control cultivars by 9 and $7 \%$, respectively, but showed high interaction with the environments and specific adaptation in low yielding environments.

Index words: Phaseolus vulgaris, diseases, genotype-environment interaction.

\section{INTRODUCCIÓN}

El frijol (Phaseolus vulgaris L.) de grano negro tiene alta demanda comercial en el sureste de México, con un consumo cercano a 300,000 $\mathrm{t}$ anuales (SIAP, 2006). En esta región, durante los últimos 10 años se sembraron en promedio 226,011 ha con este tipo de frijol, de las que se obtuvieron 133,329 t de grano, que sólo representan $42.5 \%$ de las necesidades de consumo (SAGARPA, 2012). Esto ha obligado a importar grano de otras regiones del país y del extranjero, para asegurar el abasto regional (Borja-Bravo y GarcíaSalazar, 2008).

Las principales entidades federativas productoras de frijol de la región tropical húmeda del sureste de México son Chiapas y Veracruz, con una producción promedio en los 
últimos 10 años de 97,841 t de grano, obtenidas en una superficie de 164,732 ha, con un rendimiento medio de 594 $\mathrm{kg} \mathrm{ha}^{-1}$ (SAGARPA, 2012). El rendimiento nacional en 2013 fue de $740 \mathrm{~kg} \mathrm{ha}^{-1}$ (SIAP, 2014), superior al de la región tropical húmeda. El bajo rendimiento regional es el resultado de la incidencia de diversos factores bióticos y abióticos adversos, entre los que destacan: errática distribución de las lluvias con sequía intra-estival en siembras de temporal (secano) y sequía terminal al final del ciclo, cuando el cultivo se establece bajo condiciones de humedad residual (López et al., 2002); la incidencia de enfermedades como el virus del mosaico amarillo dorado del frijol (BGYMV) (Villar et al., 2003), la roya [Uromyces appendiculatus var. appendiculatus (Pers.) Unger], la antracnosis [Colletotrichum lindemuthianum (Sacc. \& Magnus) Lams.-Scrib.] y la mancha angular [Phaeoisariopsis griseola (Sacc.) Ferraris] (López et al., 2006); así como la siembra de materiales criollos e introducidos de bajo potencial de rendimiento y susceptibles a enfermedades y a deficiencias de humedad (Tosquy et al., 2013).

En esta región el uso de semilla certificada de frijol es bajo, ya que solamente hay dos empresas dedicadas a la producción y venta de semilla de frijol, una en el Sur de Tamaulipas y otra en Chiapas. Las nuevas variedades mejoradas se han dado a conocer y dispersado a través de parcelas demostrativas establecidas en campos de productores (López et al., 2007; López et al., 2012) y de programas de multiplicación y distribución de semilla con el apoyo de presidencias municipales (López et al., 2000), de donde se inicia la dispersión de las variedades mejoradas entre los productores de la localidad y localidades aledañas.

La generación de variedades con alto potencial de rendimiento, amplia adaptación y tolerancia a los principales factores bióticos y abióticos, es una alternativa viable y económica para contribuir a solucionar la problemática antes señalada, y es también uno de los objetivos del Programa de Mejoramiento de Frijol del Instituto Nacional de Investigaciones Forestales, Agrícolas y Pecuarias (INIFAP) para el trópico húmedo. Para tal propósito se evalúan líneas y nuevas variedades en diferentes ambientes y condiciones de humedad, que permitan identificar genotipos sobresalientes, con características agronómicas superiores y mayor productividad que las de uso actual (López et al., 2012).

En la selección de mejores genotipos, es necesario considerar sus características agronómicas superiores, el rendimiento y su adaptación. Una vía para determinar la adaptación de genotipos en diferentes ambientes es mediante estudios de estabilidad del rendimiento (López et al., 2011), para lo cual se han utilizado modelos univariados (Eberhart y Russell, 1966; Cruz et al., 1989) y el modelo AMMI (análisis de efectos principales aditivos e interacción multiplicati- va) (Crossa et al., 1990). Este modelo combina una técnica estadística univariada (el modelo de Finlay y Wilkinson, 1963), con una multivariada [el análisis por componentes principales $(\mathrm{CP})$ para estudiar la respuesta de una variable en varios ambientes (CP1)]. De esta manera se genera un análisis de varianza del modelo AMMI, y un gráfico de doble entrada ("biplot" CP1 vs. rendimiento), que facilitan la interpretación de la interacción genotipo-ambiente.

El objetivo del presente estudio fue determinar el rendimiento y su estabilidad de 12 genotipos de frijol de grano negro en 12 combinaciones sitio-sistema de producción que incluyeron tres condiciones de humedad: humedad residual, temporal (secano) y riego, en los estados de Veracruz y Chiapas; así como identificar líneas experimentales que superen a los testigos comerciales en rendimiento $\mathrm{u}$ alguna otra característica agronómica ventajosa.

\section{MATERIALES Y MÉTODOS}

Se sembró un ensayo élite de rendimiento (EER) que incluyó 10 líneas avanzadas de frijol negro: SCN 2, SNC 3, SCN 4, SCN 6, NCB 229, SEN 26 y SEN 70 (provenientes del Centro Internacional de Agricultura Tropical), y ELS-15-55, NGO 17-99 y NGO 07022 del INIFAP. Los testigos fueron las variedades Negro Papaloapan y Negro Comapa. El ensayo se estableció en 12 ambientes de producción; algunos ambientes fueron en la misma localidad en diferente fecha de siembra o condición de humedad. Nueve ambientes se ubicaron en el estado de Veracruz: tres en Rincón Grande, Orizaba ( $\left.18^{\circ} 51^{\prime} \mathrm{N}, 97^{\circ} 06^{\prime} \mathrm{O}\right)$, tres en Martínez de la Torre $\left(20^{\circ} 09^{\prime} \mathrm{N}, 97^{\circ} 04^{\prime} \mathrm{O}\right)$, dos en El Laurel, San Andrés Tuxtla $\left(18^{\circ} 27^{\prime} \mathrm{N}, 95^{\circ} 10^{\prime} \mathrm{O}\right)$, y uno en Medellín de Bravo ( $18^{\circ} 50^{\prime}$ $\left.\mathrm{N}, 96^{\circ} 10^{\prime} \mathrm{O}\right)$. Los otros tres ambientes se establecieron en Ocozocoautla, Chiapas ( $\left.16^{\circ} 46^{\prime} \mathrm{N}, 93^{\circ} 22^{\prime} \mathrm{O}\right)$ (Cuadro 1).

Los genotipos se sembraron en los años 2011 y 2012 a una densidad de 250,000 plantas ha ${ }^{-1}$; se utilizó el diseño experimental de bloques completos al azar con cuatro repeticiones y parcelas experimentales de dos surcos de $5 \mathrm{~m}$ de longitud, espaciados a $0.60 \mathrm{~m}$. Todos los genotipos fueron de hábito de crecimiento tipo II y aptitud competitiva similar. El manejo agronómico se hizo de acuerdo con las recomendaciones para cultivar frijol en los estados de Veracruz (López et al., 1994) y Chiapas (Villar et al., 2002). En todas las localidades, con excepción de Rincón Grande en Orizaba, se dispuso de una estación meteorológica de la cual se obtuvo información climática en el ciclo del cultivo, principalmente precipitación. En Rincón Grande se utilizó la información de la estación No. 76,739 de la Comisión Nacional del Agua localizada en Orizaba, Ver.

En el transcurso de la estación de crecimiento hubo incidencia natural de roya en el ciclo de verano de 2011 y de 
Cuadro 1. Características de las localidades donde se condujo el Ensayo Élite de Rendimiento en los estados de Veracruz y Chiapas, México.

\begin{tabular}{lccccc}
\hline Localidad/municipio/estado & Ciclo/año & $\begin{array}{c}\text { Condición de } \\
\text { humedad }\end{array}$ & $\begin{array}{c}\text { Fecha de } \\
\text { siembra }\end{array}$ & Tipo de suelo & $\begin{array}{c}\text { Altitud } \\
(\mathrm{m})\end{array}$ \\
\hline R. Grande, Orizaba, Ver. & $\mathrm{V}^{\dagger} / 2011$ & Temporal & 26 de agosto & Migajón arenoso & 1248 \\
Medellín, Ver. & $\mathrm{O}-\mathrm{I}^{\dagger \dagger} / 2011$ & $\mathrm{HR}^{\vee}$ & 29 de septiembre & Franco & 15 \\
Martínez de la Torre, Ver. & $\mathrm{O}-\mathrm{I} / 2011-12$ & $\mathrm{HR}$ & 28 de octubre & Migajón arenoso & 92 \\
El Laurel, San Andrés Tuxtla, Ver. & $\mathrm{O}^{\dagger} \mathrm{I}^{\dagger} / 2011-12$ & $\mathrm{HR}$ & 16 de diciembre & Franco & 84 \\
Martínez de la Torre, Ver. & $\mathrm{I}^{\dagger \dagger} / 2012$ & $\mathrm{HR}$ & 17 de febrero & Migajón arenoso & 92 \\
R. Grande, Orizaba, Ver. & $\mathrm{V}^{\dagger} / 2012$ & Temporal & 03 de agosto & Migajón arenoso & 1248 \\
R. Grande, Orizaba, Ver. & $\mathrm{O}-\mathrm{I} / 2012$ & $\mathrm{HR}$ & 13 de septiembre & Migajón arenoso & 1248 \\
El Laurel, San Andrés Tuxtla, Ver. & $\mathrm{O}-\mathrm{I} / 2012-13$ & $\mathrm{HR}$ & 07 de noviembre & Franco & 84 \\
Martínez de la Torre, Ver. & $\mathrm{O}-\mathrm{I} / 2012-13$ & $\mathrm{HR}$ & 19 de octubre & Migajón arenoso & 92 \\
Ocozocoautla, Chis. & $\mathrm{O}-\mathrm{I} / 2011-12$ & $\mathrm{HR}$ & 29 de septiembre & Arcilloso & 864 \\
Ocozocoautla, Chis. & $\mathrm{I}-\mathrm{P}^{\dagger \dagger} / 2012$ & Riego & 11 de enero & Arcilloso & 864 \\
Ocozocoautla, Chis. & $\mathrm{V} / 2012$ & Temporal & 01 de mayo & Arcilloso & 864 \\
\hline
\end{tabular}

${ }^{\dagger}$ Verano. ${ }^{\dagger \dagger}$ Otoño-invierno. ${ }^{\dagger \dagger \dagger}$ Invierno-primavera. ${ }^{\vee}$ Humedad residual.

antracnosis en verano y otoño-invierno 2012 en Rincón Grande; en el Laurel, en otoño-invierno-2011-12 también hubo incidencia de antracnosis. La incidencia de las enfermedades se evaluó mediante la escala de 1.0 a 9.0 (CIAT, 1987), donde la reacción de 1 a 3 significa resistente, de 4 a 6 intermedia y de 7 a 9 susceptible. La cosecha se hizo cuando las vainas de las plantas estaban secas y el grano tenía entre 14 y $16 \%$ de humedad. El grano cosechado de cada parcela se limpió, se pesó, se le determinó su humedad y se transformó a kilogramos por hectárea a 14 \% de humedad.

Los datos de rendimiento se analizaron en forma individual y combinada; la comparación de medias fue mediante la Diferencia Mínima Significativa (DMS, $\alpha=0.05$ ). Los valores de incidencia de cada enfermedad se correlacionaron con el rendimiento de grano. Se utilizó el modelo AMMI para clasificar los ambientes e identificar líneas sobresalientes por su rendimiento de grano y menor interacción con el ambiente (Gauch y Zobel, 1996). Para el análisis de varianza y los parámetros de estabilidad se utilizó el programa de cómputo SAS® (SAS Institute, 1999), y en el desarrollo del análisis AMMI se siguieron las recomendaciones de Vargas y Crossa (2000).

\section{RESULTADOS Y DISCUSIÓN}

\section{Rendimiento y reacción a enfermedades}

Se detectaron diferencias significativas $(P \leq 0.01)$ entre ambientes, genotipos y en la interacción de ambos factores. En principio se corroboró que las características de suelo y clima (Cuadro 1) de los ambientes de prueba confirieron diferente potencial productivo a los genotipos. Por otra parte, aunque todos los genotipos pertenecen a la raza Mesoamericana (Singh et al., 1991), su resistencia a estreses abióticos y bióticos es diferente porque provienen de progenitores con características diversas y fueron seleccionados en distintas regiones. La interacción significativa genotipo $\mathrm{x}$ ambiente obedeció a la respuesta diferencial de los genotipos ante los cambios del ambiente de evaluación.

En las localidades de Martínez de la Torre, Ver., ciclo O-I 2012-2013 (1900 kg ha-1) y Ocozocoautla, Chis., O-I 2011 (1724 kg ha-1), ambas en condiciones de humedad residual, se obtuvieron los mayores rendimientos promedio (Cuadro 2), debido principalmente a que el cultivo contó con humedad almacenada del suelo, una adecuada distribución de la lluvia durante su ciclo de desarrollo (221 y $384 \mathrm{~mm}$, respectivamente) y no hubo incidencia de enfermedades. En los tres ciclos de siembra de Ocozocuatla, cada uno con diferente régimen de humedad, las condiciones climáticas fueron favorables, lo que ocasionó un alto rendimiento.

Por el contrario, en Martínez de la Torre, Ver. ciclo I-P 2012 (605 kg ha $\left.{ }^{-1}\right)$ y San Andrés Tuxtla, Ver. ciclo O-I 2011$2012\left(680 \mathrm{~kg} \mathrm{ha}^{-1}\right)$, también conducidos con humedad residual, se obtuvieron los más bajos rendimientos. En la primera localidad, aunque se tuvo una precipitación pluvial total en el ciclo de $691 \mathrm{~mm}$, su distribución fue deficiente porque hubo dos periodos sin lluvia, uno de $27 \mathrm{~d}$ que abarcó las etapas de prefloración y floración, cuando el cultivo es más susceptible a la falta de humedad (Acosta-Díaz et al., 2004), y otro de 21 d que abarcó las etapas de llenado de vainas e inicio de maduración. En la segunda localidad, el bajo 
Cuadro 2. Rendimiento promedio de genotipos de frijol negro evaluados en 12 ambientes de Veracruz y Chiapas.

\begin{tabular}{|c|c|c|c|c|c|c|}
\hline \multirow{2}{*}{ Genotipos } & \multicolumn{6}{|c|}{ Localidades de prueba } \\
\hline & 1 & 2 & 3 & 4 & 5 & 6 \\
\hline SCN 4 & 1338 & 669 & 1620 & 1317 & $838^{*}$ & 1500 \\
\hline SCN 6 & 919 & $852^{*}$ & $1760^{*}$ & 1089 & 506 & $1828^{*}$ \\
\hline SCN 3 & 956 & 724 & $1711^{*}$ & 1218 & 500 & 1388 \\
\hline NCB 229 & 494 & 675 & $1787^{*}$ & 1097 & 496 & 1509 \\
\hline SEN 26 & 676 & $816^{*}$ & 1473 & $1891^{*}$ & 594 & 1393 \\
\hline SEN 70 & 1081 & 665 & 1648 & $1766^{*}$ & $739^{*}$ & 1326 \\
\hline SCN 2 & 1088 & $873^{*}$ & $1857^{\star}$ & 1554 & $674^{*}$ & 1454 \\
\hline ELS-15-55 & 1288 & $882^{*}$ & $1763^{*}$ & 1282 & $706^{*}$ & 1513 \\
\hline NGO 07022 & 1575 & $828^{*}$ & $1959^{*}$ & 1471 & $749^{*}$ & 1509 \\
\hline NGO 17-99 & $2106^{*}$ & $813^{*}$ & 1393 & $1626^{*}$ & $897^{\star}$ & 1513 \\
\hline N Comapa & 1400 & $967^{\star}$ & $1776^{*}$ & 987 & $690^{*}$ & 1619 \\
\hline N Papaloapan & 1244 & 702 & $1939^{*}$ & 1116 & $776^{*}$ & 1578 \\
\hline Promedio & $1180 \mathrm{EF}^{\sqrt{ }}$ & $789 \mathrm{G}$ & $1724 \mathrm{AB}$ & $1368 \mathrm{CDE}$ & $680 \mathrm{G}$ & $1511 \mathrm{BC}$ \\
\hline ANVA & $* *$ & $* *$ & * & $* *$ & $* *$ & $* *$ \\
\hline CV (\%) & 21 & 14 & 12 & 15 & 23 & 9 \\
\hline DMS( 0.05) & 360.4 & 155.5 & 294.5 & 301.7 & 300.0 & 191.5 \\
\hline
\end{tabular}

\begin{tabular}{|c|c|c|c|c|c|c|c|}
\hline & & & & & & & \\
\hline SCN 4 & 491 & $1504^{*}$ & $1839^{*}$ & 1144 & 1348 & 1699 & $1275 \mathrm{abc}$ \\
\hline SCN 6 & 450 & $1567^{\star}$ & 603 & 225 & 1061 & 1680 & $1045 \mathrm{~d}$ \\
\hline $\mathrm{SCN} 3$ & 477 & $1577^{\star}$ & 1384 & 699 & 1253 & 1771 & $1138 \mathrm{bcd}$ \\
\hline NCB 229 & 679 & $1691^{\star}$ & 964 & 751 & 1081 & $1886^{*}$ & $1093 \mathrm{~cd}$ \\
\hline SEN 26 & 791 & $1463^{*}$ & 851 & 920 & 1183 & $2289^{\star}$ & $1195 \mathrm{bcd}$ \\
\hline SEN 70 & $1054^{\star}$ & $1551^{\star}$ & $2133^{*}$ & $1931^{\star}$ & 1196 & $2151^{\star}$ & 1437 a \\
\hline SCN 2 & $931^{\star}$ & $1483^{\star}$ & 1478 & $1679^{*}$ & 1229 & $1861^{\star}$ & $1347 \mathrm{ab}$ \\
\hline ELS-15-55 & 260 & 1345 & 878 & 489 & 1304 & 1378 & $1091 \mathrm{~cd}$ \\
\hline NGO 07022 & 512 & 1104 & $1695^{\star}$ & 1103 & 1425 & $1901^{\star}$ & $1319 \mathrm{ab}$ \\
\hline NGO 17-99 & 616 & 1287 & $1685^{\star}$ & 1118 & 1342 & $1827^{\star}$ & $1352 \mathrm{ab}$ \\
\hline N Comapa & 544 & 1324 & $2035^{\star}$ & 1311 & 1244 & $2155^{\star}$ & $1338 \mathrm{ab}$ \\
\hline N Papaloapan & 456 & 1326 & 1189 & $1848^{\star}$ & 1311 & $2199^{*}$ & $1307 \mathrm{abc}$ \\
\hline Promedio & $605 \mathrm{G}$ & $1435 \mathrm{CD}$ & $1394 \mathrm{CDE}$ & $1101 \mathrm{~F}$ & $1248 \mathrm{DEF}$ & $1900 \mathrm{~A}$ & 1245 \\
\hline ANVA & $* *$ & * & $* *$ & $* *$ & ns & * & $* *$ \\
\hline CV (\%) & 17 & 14 & 23 & 20 & 13 & 17 & 17 \\
\hline DMS( 0.05$)$ & 148.1 & 282.4 & 463.6 & 320.9 & & 463.3 & 219.72 \\
\hline
\end{tabular}


rendimiento se debió a dos factores: a) La incidencia de antracnosis (Cuadro 3), que disminuyó significativamente el rendimiento $\left(r=-0.70^{\star}\right)$, provocada por un exceso de humedad (106 $\mathrm{mm}$ de precipitación pluvial) y temperaturas templadas entre 14 y $25^{\circ} \mathrm{C}$ durante las etapas de inicio de floración y formación de vainas; $\mathrm{y} b$ ) La presencia de un periodo sin lluvias a partir del inicio de llenado de vainas hasta la cosecha, en donde sólo se registraron $10 \mathrm{~mm}$ de lluvia (CNA, 2013).

En Orizaba, Ver., la presencia de roya en el temporal (secano) de 2011 y de antracnosis en O-I 2012 con humedad residual (Cuadro 3), causaron disminución del rendimiento $\left(\mathrm{r}=-0.84^{* *}\right.$ y $-0.70^{*}$, respectivamente). En el ciclo de verano de 2012, la antracnosis (Cuadro 3) no afectó significativamente el rendimiento $(r=-0.52 \mathrm{~ns})$. Estos resultados demuestran la importancia de evaluar a los genotipos en varios ciclos y años, aún en una misma localidad, para determinar con mayor precisión la influencia de factores abióticos y bióticos en el cultivo de frijol, como ya lo apuntaban López et al. (2006).

En promedio de ambientes, las líneas más productivas fueron SEN 70, NGO 17-99 y SCN 2, cuyo rendimiento promedio fue estadísticamente similar al de líneas SCN 4 y NG 07022 y los testigos regionales (Papaloapan y Comapa) (Cuadro 2). En cada ambiente de prueba diferentes líneas ocuparon el primer sitio en rendimiento, y en seis de 12 ambientes la línea de mayor rendimiento fue superior a los testigos $(\mathrm{P}<0.05)$. La línea SEN 70, seleccionada por su resistencia a la sequía en Colombia (Beebe et al., 2008), ocupó el primer sitio en tres ambientes, y NGO 17-99 en dos. Los resultados confirmaron que los testigos Comapa y Papaloapan, de reciente registro para el estado de Veracruz, son resistentes a enfermedades (López-Salinas et al., 2007; López et al., 2010), son similares en rendimiento en la mayoría de los ambientes, con excepción de Verano $2012 \mathrm{~T}$ y OI 2012 en Orizaba, Ver., en los que mostraron respuesta contrastante.

Los genotipos de mayor rendimiento mostraron resistencia a la antracnosis que incidió en San Andrés Tuxtla en el ciclo O-I 2011-12 y en Orizaba, en el ciclo O-I 2012 (Cuadro 3); sin embargo, SEN 70 y SCN 2 mostraron moderada susceptibilidad a la roya que se presentó en el ciclo de temporal 2011 en Orizaba, mientras que NGO 17-99 fue resistente. En contraste, las líneas de menor rendimiento SCN 6, ELS 15-55 y NCB 229 fueron susceptibles a las enfermedades. En el caso de las líneas introducidas del CIAT, su característica principal es la resistencia a la sequía y su adaptación en ambientes de alta productividad y a suelos de bajo fósforo (Beebe et al., 2008). La línea SEN 70 ejemplifica lo anterior, pues en Martínez de la Torre I-P 2012 (el ambiente de menor rendimiento) superó a los testigos en $100 \%$, y en el ambiente de mayor rendimiento (Martínez de la Torre O-I 2012) mostró un rendimiento similar al de éstos (Cuadro 2).

Cuadro 3. Reacción de 12 genotipos de frijol negro a enfermedades (escala 1 a 9) presentes en cuatro ambientes de Veracruz.

\begin{tabular}{|c|c|c|c|c|}
\hline Genotipo & Roya $^{1}$ & Antracnosis $^{2}$ & Antracnosis $^{3}$ & Antracnosis ${ }^{4}$ \\
\hline SCN 4 & 1.50 & 2.50 & $3.25^{*}$ & 2.00 \\
\hline SCN 6 & 4.50 & $4.00 *$ & $4.50 *$ & $7.50 *$ \\
\hline SCN 3 & 3.50 & $4.25^{\star}$ & $4.25^{*}$ & 6.25 \\
\hline NCB 229 & $6.75^{*}$ & 2.75 & $2.75^{*}$ & 2.00 \\
\hline SEN 26 & 4.50 & $5.00 *$ & $4.75^{*}$ & 5.75 \\
\hline SEN 70 & 5.00 & 1.00 & 1.00 & 1.50 \\
\hline SCN 2 & 4.75 & 1.25 & 1.25 & 1.25 \\
\hline ELS 15-55 & 2.00 & 1.50 & 2.50 * & 3.00 \\
\hline Ng 07022 & 1.75 & 1.00 & 2.00 & 1.25 \\
\hline Ng 17-99 & 1.25 & 1.25 & $2.75^{*}$ & 4.25 \\
\hline Negro Comapa & 1.75 & 1.50 & 2.25 & 1.75 \\
\hline Negro Papaloapan & 2.00 & 1.00 & 1.00 & 1.50 \\
\hline Promedio & 3.27 & 2.25 & 2.69 & 3.17 \\
\hline DMS (0.05) & 1.175 & 1.184 & 2.44 & 1.17 \\
\hline
\end{tabular}




\section{Análisis AMMI}

El análisis mostró que los cinco primeros componentes principales presentaron variabilidad significativa y acumularon $95 \%$ de la varianza; de estos cinco, los primeros tres explicaron $82.1 \%$ de la suma de cuadrados y los dos primeros explicaron $70 \%$ (Cuadro 4), lo cual se considera satisfactorio (Santos-Pereira et al., 2009).

Los ambientes correspondientes a Martínez de la Torre, Ver., ciclos I-P 2012 y O-I 2012-13 mostraron la menor interacción con los genotipos (CP $1=-1.5324$ y -0.2399 , respectivamente) y el menor y mayor rendimiento promedio (Cuadro 4, Figura 1). Cabe indicar que San Andrés Tuxtla y Martínez de la Torre, por ser localidades de baja interacción con los genotipos, son ideales para identificar materiales estables para las zonas centro y norte de Veracruz y con posibilidades de adaptación en otras áreas con características ambientales similares. Estas localidades se utilizaron en el proceso de selección y evaluación de los testigos (LópezSalinas et al., 2007; López et al., 2010), cuya resistencia a enfermedades y potencial de rendimiento fue evidente en este estudio.

En Orizaba, Ver., ciclos V 2012 y O-I 2012, se observó la interacción más alta entre el ambiente y los genotipos (CP 1 $=25.2234$ y 25.7814, respectivamente) (Cuadro 4, Figura 1), lo cual se atribuye principalmente a la incidencia de antracnosis en ambos ciclos de producción, y donde los genotipos mostraron diferente reacción a esta enfermedad (Cuadro 3). La línea SCN 6 mostró el más bajo rendimiento en esos dos ambientes, ya que fue dañada por la antracnosis, seguida de SEN 26 y SCN 3. El resto de los genotipos mostró tolerancia a la enfermedad. En esta localidad también se ha observado incidencia del patógeno causante de la mancha angular (Tosquy et al., 2013), por lo que es apta para la selección por resistencia múltiple a enfermedades. En Ocozocoautla, Chis. se obtuvieron altos rendimientos promedio de grano, pero durante los ciclos I-P 2012 y V 2012 se observó alta interacción del ambiente con los genotipos, aunque no de la magnitud observada en Córdoba, Ver. (Figura 1).

Siete genotipos se ubicaron en el cuadrante superior derecho que fue el de mayor rendimiento (Figura 2); de éstos, NGO 07022 y SCN 2 mostraron la menor interacción con el ambiente con CP 1 de 5.7497 y 6.8491, respectivamente (Cuadro 5). La estabilidad de estos dos genotipos está relacionada con su resistencia a enfermedades y a la sequía terminal (Beebe et al., 2008). El testigo regional Negro Papaloapan también obtuvo alto rendimiento promedio y

Cuadro 4. Resultados de la suma de cuadrados en términos $\mathrm{AMMI}^{\dagger}$.

\begin{tabular}{|c|c|c|c|c|c|}
\hline \multirow{2}{*}{ Fuente } & \multirow{2}{*}{ GLAMMI } & \multirow{2}{*}{ SCAMMI } & \multirow{2}{*}{ CMAMMI } & \multicolumn{2}{|c|}{ Explicación de la varianza (\%) } \\
\hline & & & & Individual & Acumulada \\
\hline Repeticiones & 3 & $438,236.14$ & $146,078.71^{*}$ & & \\
\hline Ambientes (A) & 11 & $84,634,225.47$ & $7,694,020.50^{* *}$ & & \\
\hline Genotipos (G) & 11 & $8,562,138.43$ & $778,376.22^{\star *}$ & & \\
\hline$(\mathrm{G} \times \mathrm{A})$ & 121 & $36,493,473.82$ & $301,598.96^{* *}$ & & \\
\hline $\mathrm{CP} 1$ & 21 & $15,417,608.30$ & $734,171.83^{\star *}$ & 42.2 & 42.2 \\
\hline $\mathrm{CP} 2$ & 19 & $10,128,253.80$ & $533,065.99^{* *}$ & 27.8 & 70.0 \\
\hline $\mathrm{CP} 3$ & 17 & $4,411,969.27$ & $259,527.60^{\star *}$ & 12.1 & 82.1 \\
\hline $\mathrm{CP} 4$ & 15 & $3,224,243.81$ & $214,949.59^{\star \star}$ & 8.8 & 90.9 \\
\hline CP5 & 13 & $1,496,205.05$ & $115,092.70^{* *}$ & 4.1 & 95.0 \\
\hline CP6 & 11 & $890,534.41$ & $80,957.67$ & 2.4 & 97.5 \\
\hline $\mathrm{CP} 7$ & 9 & $550,639.00$ & $61,182.11$ & 1.5 & 99.0 \\
\hline СР8 & 7 & $194,504.72$ & $27,786.39$ & 0.5 & 99.5 \\
\hline СР9 & 5 & $133,607.96$ & $26,721.59$ & 0.4 & 99.9 \\
\hline CP10 & 3 & $37,709.96$ & $12,569.99$ & 0 & 100 \\
\hline CP11 & 1 & 8197.56 & 8197.56 & 0 & 100 \\
\hline Error & 429 & $20,969,786.40$ & $48,880.60$ & & \\
\hline $\mathrm{CV}(\%)=18$ & & $\mathrm{R}^{2}=0.86$ & & & \\
\hline
\end{tabular}

${ }^{\dagger}$ Efectos principales aditivos e interacción multiplicativa; GLAMMI = Grados de libertad. SCAMMI = Suma de cuadrados. CMAMMI = Cuadrados medios. CP $=$ Componente principal. ${ }^{*}=\mathrm{P} \leq 0.05,{ }^{* *}=\mathrm{P} \leq 0.01$. 


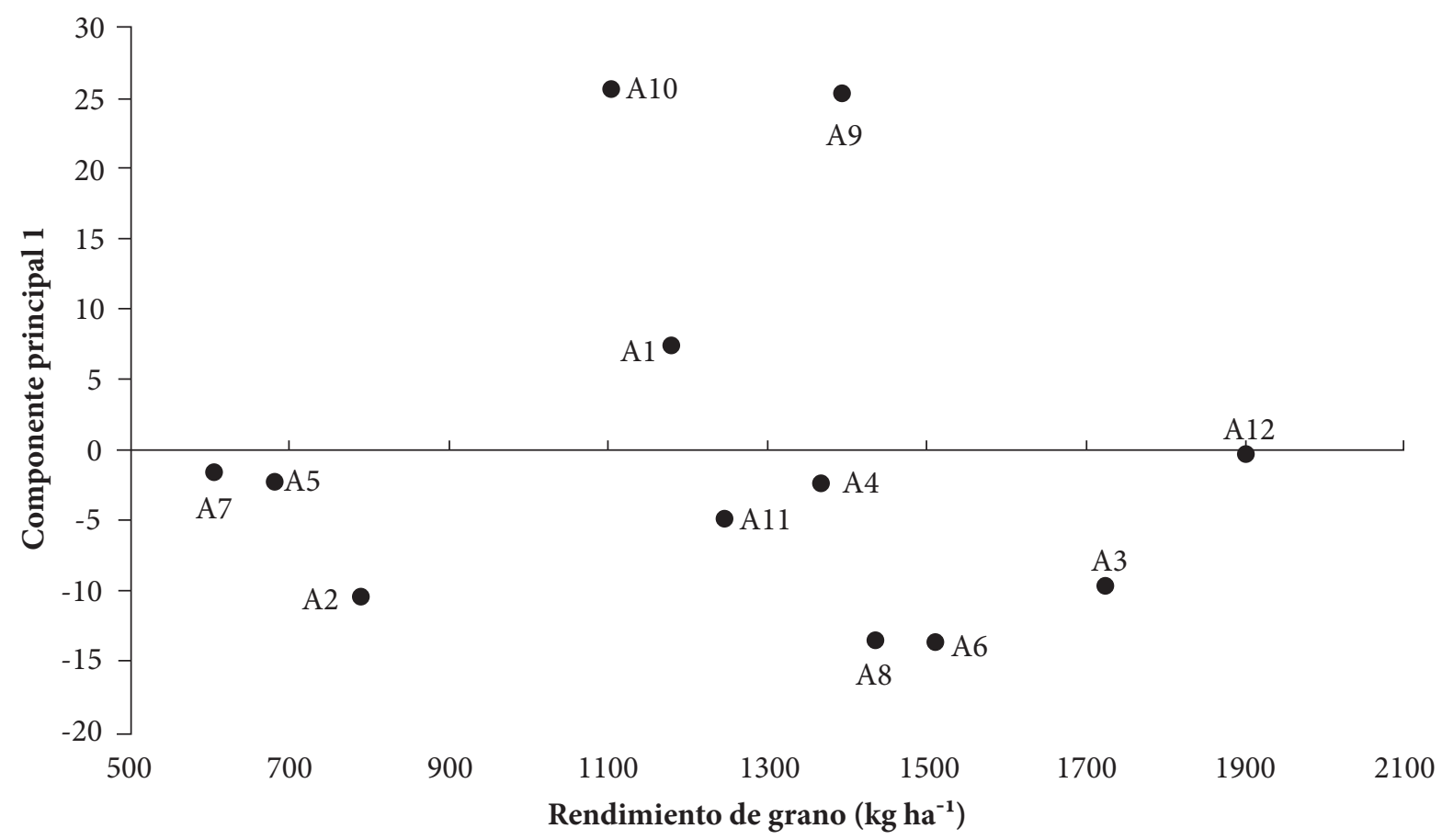

Figura 1. Efectos principales e interacción observada para 12 ambientes de prueba. A1: Orizaba, Ver., V 2011. A2: Medellín, Ver., O-I 2011. A3: Ocozocoautla, Chis., O-I 2011. A4: Martínez de la Torre, Ver., O-I 2011-12. A5: San Andrés Tuxtla, Ver., O-I 2011-12. A6: Ocozocoautla, Chis., I-P 2012. A7: Martínez de la Torre, Ver., I-P 2012. A8: Ocozocoautla, Chis., V 2012. A9: Orizaba, Ver., V 2012. A10: Orizaba, Ver., O-I 2012. A11: San Andrés Tuxtla, Ver., O-I 2012-13. A12: Martínez de la Torre, Ver., O-I 2012-13.

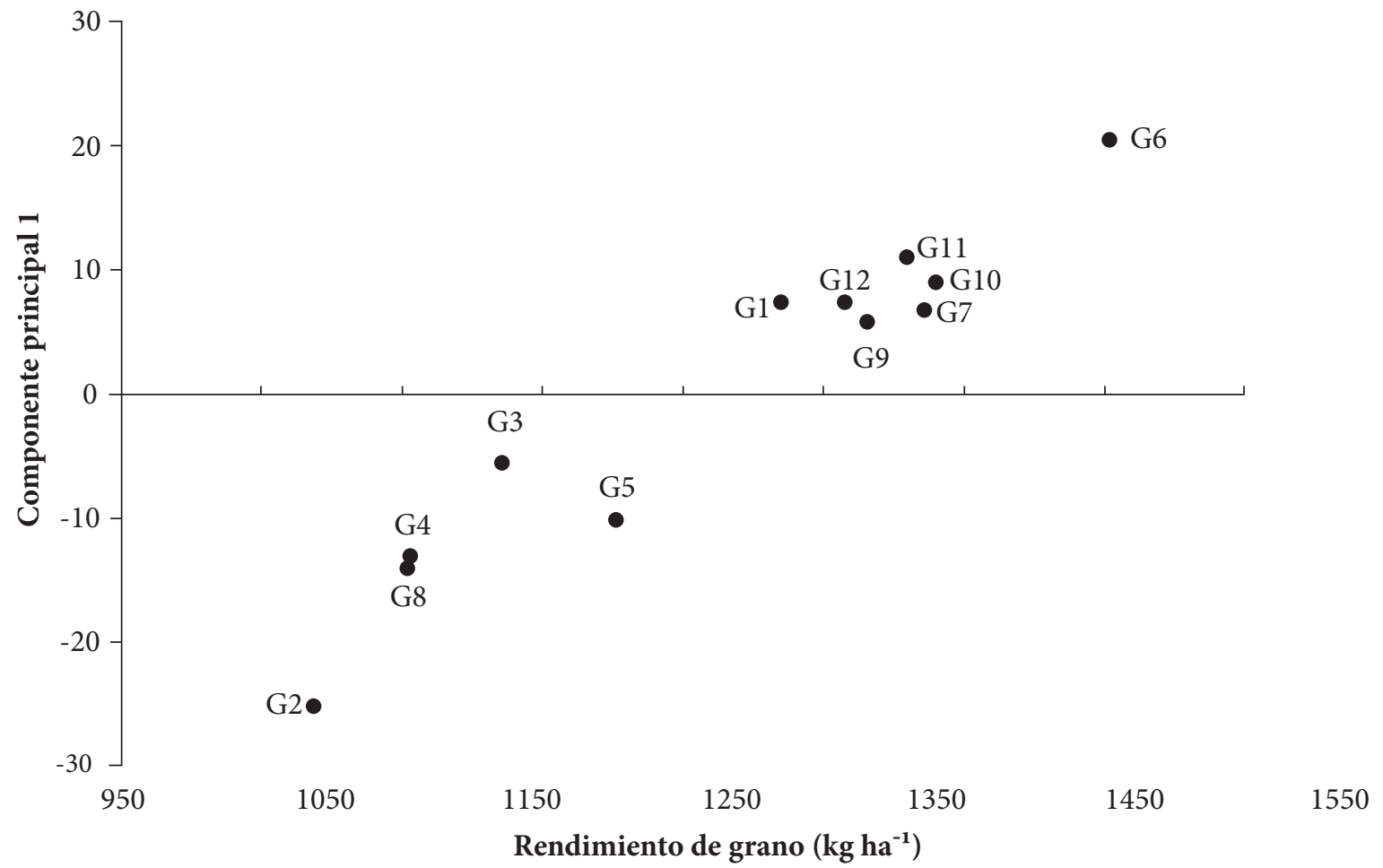

Figura 2. Efectos principales e interacción observada para el rendimiento de 12 genotipos de frijol negro. G1: SCN 4; G2: SCN 6; G3: SCN 3; G4: NCB 229; G5: SEN 26; G6: SEN 70; G7: SCN 2; G8: ELS 15-55; G9: NGO 07022; G10: NGO 17-99; G11: Negro Comapa; G12: Negro Papaloapan. 
Cuadro 5. Rendimiento promedio de genotipos, ambientes y valores de componentes principales significativos.

\begin{tabular}{|c|c|c|c|c|c|}
\hline Tipo & Genotipo/Ambiente & $\begin{array}{l}\text { Rendimiento } \\
\left(\mathrm{kg} \mathrm{ha}^{-1}\right)\end{array}$ & $\mathrm{CP} 1$ & $\mathrm{CP} 2$ & $\mathrm{CP} 3$ \\
\hline G 1 & SCN 4 & 1275 & 7.3575 & 7.3654 & -0.5621 \\
\hline G 10 & NGO 17-99 & 1352 & 9.1452 & 19.0891 & 14.9106 \\
\hline G 11 & Negro Comapa & 1338 & 11.1119 & 5.8359 & -13.4415 \\
\hline G 12 & Negro Papaloapan & 1307 & 7.2314 & -6.1816 & -15.1379 \\
\hline G 2 & SCN 6 & 1045 & -25.1543 & 3.4128 & -3.7057 \\
\hline G 3 & SCN 3 & 1138 & -5.4978 & 0.6523 & -1.6874 \\
\hline G 4 & NCB 229 & 1093 & -13.2938 & -12.9322 & -6.8785 \\
\hline G 5 & SEN 26 & 1195 & -10.1328 & -17.0504 & 17.533 \\
\hline G 6 & SEN 70 & 1437 & 20.7151 & -14.8138 & 6.5136 \\
\hline G 7 & SCN 2 & 1347 & 6.8491 & -10.1007 & 0.3604 \\
\hline G 8 & ELS 15-55 & 1091 & -14.0812 & 13.6285 & 1.9725 \\
\hline G 9 & NGO 07022 & 1319 & 5.7497 & 11.0947 & 0.123 \\
\hline A 1 & Orizaba, Ver., V 2011, Temporal & 1180 & 7.4071 & 28.3133 & 4.8948 \\
\hline A 10 & Orizaba, Ver., O-I 2012, $\mathrm{HR}^{\dagger}$ & 1101 & 25.7814 & -15.2154 & -7.4423 \\
\hline A 11 & San Andrés Tuxtla, Ver., O-I 2012-13, HR & 1248 & -4.8518 & 5.7113 & -0.5248 \\
\hline A 12 & Martínez de la Torre, Ver., O-I 2012-13, HR & 1900 & -0.2399 & -12.7597 & -1.7648 \\
\hline A 2 & Medellín, Ver., O-I 2011, HR & 789 & -10.427 & 3.5731 & -1.4975 \\
\hline A 3 & Ocozocoautla, Chis., O-I 2011, HR & 1724 & -9.5529 & -0.6116 & -14.8485 \\
\hline A 4 & Martínez de la Torre, Ver., O-I 2011-12, HR & 1368 & -2.396 & -6.1084 & 24.8903 \\
\hline A 5 & San Andrés Tuxtla, Ver., O-I 2011-12, HR & 680 & -2.1673 & 5.5681 & 1.1583 \\
\hline A 6 & Ocozocoautla, Chis., I-P 2012, Riego & 1511 & -13.6568 & 4.5206 & -7.7537 \\
\hline A 7 & Martínez de la Torre, Ver., I-P 2012, HR & 605 & -1.5324 & -12.4575 & 7.3311 \\
\hline A 8 & Ocozocoautla, Chis., V 2012, Temporal & 1435 & -13.5878 & -7.5278 & -1.9258 \\
\hline \multirow[t]{2}{*}{ A 9} & Orizaba, Ver., V 2012, Temporal & 1394 & 25.2234 & 6.9939 & -2.5172 \\
\hline & Media & 1245 & & & \\
\hline
\end{tabular}

$\mathrm{CP}=$ componentes principales. $\mathrm{G}=$ genotipo. $\mathrm{A}=$ ambiente. ${ }^{\dagger}$ Humedad residual.

reducida interacción con el ambiente, lo cual fue reportado por López et al. (2011).

Un genotipo ideal es el que tiene rendimiento alto y estable, mientras que los que presentan baja estabilidad y bajo rendimiento no son deseables (Ferreira et al., 2006); de ser posible, es importante seleccionar los que interaccionan en menor grado con el ambiente (Vargas y Crossa, 2000). Por su parte, las líneas SEN 70 y SCN 6 que obtuvieron el mayor y menor rendimiento promedio, respectivamente, mostraron la más alta interacción con el ambiente con CP 1 = 20.7151 y -25.1543 (Cuadro 5). La línea SEN 70 mostró adaptación específica en los ambientes de menor productividad en los que superó ampliamente a los testigos, y por ello se incluirá en el proceso de validación con agricultores cooperantes en el norte-centro de Veracruz. En caso de que esta línea presente estabilidad en esa área, se procederá a su registro y multiplicación de semilla a través del esquema oficial y del no-tradicional para distribución de semilla a los productores.

\section{CONCLUSIONES}

El efecto ambiental sobre el rendimiento fue mayor que el de genotipos. La línea SEN 70 presentó el mayor rendimiento promedio al superar en 7 y $9 \%$ a los testigos Comapa y Papaloapan, y mostró adaptación específica en los ambientes de menor potencial productivo. En algunos ambientes la incidencia de enfermedades disminuyó el rendimiento de los genotipos susceptibles y contribuyó al efecto ambiental y a la interacción genotipo $\mathrm{x}$ ambiente, pero también en estos casos se detectaron líneas tolerantes a enfermedades y a sequía, que superan o igualan a los testigos, como NGO 07022 y SCN 2. 


\section{AGRADECIMIENTOS}

Al fondo sectorial SAGARPA-CONACYT, por el apoyo proporcionado al proyecto 2009-109621 "Desarrollo de variedades de frijol de alto rendimiento, tolerantes a sequía, resistentes a patógenos y con la calidad que demanda al consumidor", a través del cual se financiaron las actividades de investigación de 2009 a 2013.

\section{BIBLIOGRAFÍA}

Acosta-Díaz E., C. Trejo-López, L. del M. Ruíz-Posadas, J. S. PadillaRamírez y J. A. Acosta-Gallegos (2004) Adaptación del frijol a sequía en la etapa reproductiva. Terra Latinoamericana 22:49-58.

Beebe S. E., I. M. Rao, C. Cajiao and M. Grajales (2008) Selection for drought tolerance in common bean also improves yield in phosphorus limited environments. Crop Science 48:582-592

Borja-Bravo M. y J. A. García-Salazar (2008) Políticas para disminuir las importaciones de frijol (Phaseolus vulgaris L.) en México: Un análisis por tipo de variedad. Agrociencia 42:949-958.

CIAT, Centro Internacional de Agricultura Tropical (1987) Sistema Estándar para la Evaluación de Germoplasma de Frijol. A van Schoonhoven y M A Pastor-Corrales (comps.). CIAT. Cali, Colombia. $56 \mathrm{p}$.

CNA, Comisión Nacional del Agua (2013) Servicio Meteorológico Nacional. Disponible en: Información Histórica/Climatología/ TempyPrecip/Mensuales/2012Precip.pdf. (Octubre 2014).

Crossa J., H. G. Gauch Jr. and R. W. Zobel (1990) Additive main effects and multiplicative interaction analysis of two international maize cultivar trails. Crop Science 30:493-500.

Cruz C. D., R. A. Torres and R. Vencovsky (1989) An alternative approach to the stability analyses proposed by Silva and Barreto. Revista Brasileira de Genética 12:567-580.

Eberhart S. A. and W. A. Russell (1966) Stability parameters for comparing varieties. Crop Science 6:36-40.

Ferreira D. F., C. G. B. Demetrio, B. F. J. Manly, A. A. Machado and R. Vencovsky (2006) Statistical models in agriculture: Biometrical methods for evaluating phenotypic stability in plant breeding. Cerne Lavras 12:373-388.

Finlay K. W. and G. N. Wilkinson (1963) The analysis of adaptation in a plant-breeding program. Australian Journal of Agricultural Research 14:742-754.

Gauch H. G. and R. W. Zobel (1996) AMMI analysis of yield trials. In: Genotype-by-Environment Interaction. M. S. Kang and H. G. Gauch (eds.). CRC Press. Boca Raton, FL, USA. pp:1-40.

López Salinas E., A. Durán Prado, E. N. Becerra Leor, V. A. Esqueda Esquivel y O. Cano Reyes (1994) Manual de Producción de Frijol en el Estado de Veracruz. Folleto para Productores No. 7. SARH. INIFAP. CIRGOC. Campo Experimental Cotaxtla. Cotaxtla. Veracruz, México. $29 \mathrm{p}$

López Salinas E., J. A. Acosta Gallegos, O. H. Tosquy Valle, R. A. Salinas Pérez, B. M. Sánchez García, R. Rosales Serna, C. González Rivas, T. Moreno Gallegos, B. Villar Sánchez, H. M Cortinas Escobar y R. Zandate Hernández (2011) Estabilidad de rendimiento en genotipos mesoamericanos de frijol de grano negro en México. Revista Mexicana de Ciencias Agrícolas 2:29-40.
López Salinas E., E. N. Becerra Leor, O. Cano Reyes y V. O. López Galván (2002) Detección de líneas y variedades de frijol (Phaseolus vulgaris $\mathrm{L}$.) con resistencia múltiple a enfermedades en el trópico húmedo de México. Revista Mexicana de Fitopatología 20:193-199.

López Salinas E., O. H. Tosquy Valle, B. Villar Sánchez, E. N. Becerra Leor, F. J. Ugalde Acosta y J. Cumpián Gutiérrez (2006) Adaptabilidad de genotipos de frijol resistentes a enfermedades a y suelos ácidos. Revista Fitotecnia Mexicana 29:33-39.

López Salinas E., O. H. Tosquy Valle, B. Villar Sánchez, J. Cumpián Gutiérrez, F. J. Ugalde Acosta y E. N. Becerra Leor (2007) Negro Papaloapan, nuevo cultivar de frijol para las áreas tropicales de México. Agricultura Técnica en México 33:257-267.

López Salinas E., O. H. Tosquy Valle, B. Villar Sánchez, J. R. Rodríguez Rodríguez, F. J. Ugalde Acosta, A. Morales Rivera y J. A. Acosta Gallegos (2010) Negro Comapa, nueva variedad de frijol para el estado de Veracruz. Revista Mexicana de Ciencias Agrícolas 1:715-721.

López Salinas E., O. H. Tosquy Valle, Y. Jiménez Hernández, R. A. Salinas Pérez, B. Villar Sánchez y J. A. Acosta Gallegos (2012) Rendimiento y adaptación de la variedad de frijol Negro Comapa en dos regiones de México. Revista Fitotecnia Mexicana 35:309-315.

Santos-Pereira H., L. Cunha-Melo, L. C. de Faria, J. L. Cabrera-Díaz, M. J. del Peloso, J. G. Cáprio da Costa and A. Wendland (2009) Stability and adaptability of carioca common bean genotypes in states of the central South Region of Brazil. Crop Breeding and Applied Biotechnology 9:181-188

SAGARPA, Secretaría de Agricultura, Ganadería, Desarrollo Rural, Pesca y Alimentación (2012) Anuarios Estadísticos de la Producción Agrícola. Servicio de Información Agroalimentaria y Pesquera (SIAP). SAGARPA. México, D. F. http://www.siap. sagarpa.gob.mx (Julio 2012).

SIAP, Servicio de Información y Estadística Agroalimentaria y Pesquera (2006) Situación Actual y Perspectivas de la Producción de Frijol en México 2000-2005. SIAP. Secretaría de Agricultura, Ganadería, Desarrollo Rural, Pesca y Alimentación (SAGARPA). México. 34 p.

SIAP, Servicio de Información y Estadística Agroalimentaria y Pesquera (2014) Frijol, Balanza Disponibilidad-Consumo 2013. www.siap.gob.mx/wp-content/uploads/2013/balanza\%cd20\%frijol\%2022009-2013.pdf (Enero 2015).

Singh S. P., P. Gepts and D. G. Debouck (1991) Races of common bean (Phaseolus vulgaris, Fabaceae). Economic Botany 45:379-396.

SAS Institute (1999) SAS/STAT User’s Guide. Software versión 8.0. SAS Institute Inc. Cary, NC, USA.

Tosquy Valle O. H., E. López Salinas, E. N. Becerra Leor, V. A. Esqueda Esquivel y J. R. Rodríguez Rodríguez (2013) Reacción a mancha angular y productividad de genotipos de frijol con y sin aplicación de fungicidas. Revista Mexicana de Ciencias Agrícolas 4:167-174.

Vargas M. y J. Crossa (2000) El Análisis AMMI y la Gráfica del Biplot en SAS. Centro Internacional para el Mejoramiento de Maíz y Trigo (CIMMYT). México, D. F. 42 p.

Villar Sánchez B., E. R. Garrido Ramírez, A. López Luna y F. J. Cruz Chávez (2002) Manual para la Producción de Frijol en el Estado de Chiapas. Publicación Especial No. 1. SAGARPA. INIFAP. CIRPAS. Campo Experimental Centro de Chiapas. Ocozocoautla de Espinosa, Chis., México. 165 p.

Villar Sánchez B., E. López Salinas y J. Acosta Gallegos (2003) Selección de genotipos de frijol por rendimiento y resistencia al mosaico dorado y suelos ácidos. Revista Fitotecnia Mexicana 26:109-114. 